\title{
DISTRIBUIÇÃO DE POROS DE UM LATOSSOLO VERMELHO EUTROFÉRRICO NA FASE DE IMPLANTAÇÃO DE UM SISTEMA DE PLANTIO DIRETO
}

\author{
RAFFAEL A. LAURANI ${ }^{1}$, RICARDO RALISCH ${ }^{2}$, JOÃO TAVARES FILHO ${ }^{2}$, \\ DÉCIO S. SOARES ${ }^{3}$, ADRIANA A. RIBON ${ }^{4}$
}

\begin{abstract}
RESUMO: O estudo da distribuição de poros é importante na avaliação física do solo submetido a diferentes sistemas de produção. O objetivo deste trabalho foi avaliar a distribuição de poros de um Latossolo Vermelho Eutroférrico, como um parâmetro de sua qualidade física para analisar sua eficiência na implantação do sistema de plantio direto. Empregou-se um delineamento experimental inteiramente casualizado, com quatro tratamentos, na rotação de inverno (milho safrinha, coquetel de adubos verdes, aveia-preta e trigo) e duas profundidades (0-0,2 $\mathrm{m}$ e $0,2-0,4 \mathrm{~m})$, com três repetições por tratamento. Após três anos de implantação dos tratamentos (coberturas vegetais), foram realizadas determinações físicas (densidade do solo), fisico-hídricas do solo (macroporosidade, microporosidade, porosidade total e curva de retenção de água do solo) e matéria orgânica (uma amostra composta por dez repetições por tratamento). Os resultados obtidos mostraram que a utilização de milho "safrinha", coquetel de adubos verdes, aveia-preta e trigo, na rotação de inverno, não influenciou na macroporosidade do solo (entre 0 e $30 \mathrm{cca}$ ), na microporosidade, na porosidade total, na retenção de água e na densidade do solo, nas camadas de 0-0,2 e 0,2-0,4 m, e que a rotação de inverno com aveiapreta promoveu maior macroporosidade do solo (entre 0 e $60 \mathrm{cca}$ ) em relação à rotação com milho e trigo na camada de 0-0,2 m e, com trigo, na camada de 0,2-0,4 m. Para todas as rotações de inverno, os maiores valores de densidade do solo ocorrem na camada de 0-0,2 $\mathrm{m}$.
\end{abstract}

PALAVRAS-CHAVE: porosidade, curva de retenção, manejo do solo.

\section{PORE DISTRIBUTION OF A EUTROFERRIC RED LATOSOL IN THE INITIAL STAGE OF A NO TILLAGE SYSTEM}

SUMMARY: The study of pore distribution is an important physical evaluation of the soil submitted to different production systems. The purpose of this paper is to evaluate the pore distribution of a Eutroferric Red Latosol as a parameter of its physical quality to analyze its efficiency in the initiation of a no-till system. A fully randomized experimental design was used with four rotation treatments in Winter (winter maize, green cover rotation, black oats and wheat) and two depths $(0-0.2 \mathrm{~m}$ and $0.2-$ $0.4 \mathrm{~m}$ ) with three replicates per treatment. After three years of initialization of the treatments (vegetal covers) physical (bulk density), physical-hydrical (macro and micro porosity, total porosity and soil water retention curve) and organic matter (one amostre composed by ten replicates per treatment) evaluations were carried out. The results obtained showed that the vegetables covers didn't influenced the macro porosity; the micro porosity, total porosity, the water retention and the bulk density in two depths, $0-0.2$ and $0.2-0.4 \mathrm{~m}$, and, the black oat rotation promoted the biggest macro porosity of soil in a depth of $0-0.2 \mathrm{~m}$ in comparison with winter maize and wheat rotations and with wheat rotation in a depth of 0.2-0.4 m. In all treatments the biggest values of bulk density was in a depth of 0-0.20 m.

KEYWORDS: porosity, retention curve, soil management.

\footnotetext{
${ }^{1}$ Eng. Agr., Mestrando pela Universidade Estadual de Londrina, e-mail: rarelaurani@ brturbo.com

${ }^{2}$ Prof. Dr., Universidade Estadual de Londrina, Centro de Ciências Agrárias, Departamento de Agronomia, Caixa Postal 6001, Londrina -PR, e-mail: ralisch@uel.br; tavares@uel.br

${ }^{3}$ Graduando em Agronomia pela Universidade Estadual de Londrina, e-mail: decioagro@ hotmail.com

${ }^{4}$ Eng. Agr., Doutoranda pela Universidade Estadual de Londrina, e-mail: aaribon@ yahoo.com.br

Recebido pelo Conselho Editorial em: 29-4-2002

Aprovado pelo Conselho Editorial em: 27-5-2004
} 


\section{INTRODUÇÃO}

O solo é um sistema tridimensional, complexo, sendo composto por espaços porosos e sólidos. O espaço sólido de um solo é composto por material mineral e orgânico. O espaço poroso é a porção volumétrica do solo ocupada por ar e água (HILLEL, 1982). A porosidade é importante para a infiltração de água, desenvolvimento de raízes, transporte de gases e solutos, entre outros. A caracterização físico-hídrica de um solo é essencial para nortear as práticas de manejo, destacando-se irrigação, drenagem, cultivos e subsolagem (JORGE \& PRADO, 1988).

A prática tem mostrado que raramente a condição ótima de porosidade é alcançada no solo, devido a influências de manejo exercidas pelo homem. A compactação, por exemplo, induz o aumento de densidade do solo, diminuição da porosidade total e alteração na distribuição dos tamanhos dos poros (HORTON et al., 1994).

A porosidade total e a sua distribuição pelo tamanho são características físicas do solo indiretamente relacionadas à estrutura e podem ser avaliadas pela densidade e macroporosidade do solo (CORSINI \& FERRAUDO, 1999).

A distribuição do tamanho dos poros é mais importante para a curva característica (curva de retenção de água) próximo à saturação. Conforme decresce a umidade, os filmes de água ao redor de agregados dominam a curva característica. Assim, em solos agregados, não-deformados, a agregação domina a retenção de água para altos conteúdos de água enquanto a textura e a mineralogia se tornam mais importantes para baixos conteúdos de água (RAWLS et al., 1991).

No sistema de plantio direto, a porosidade total do solo é menor quando comparada ao sistema convencional, porém há maior proporção de macroporos. Esse aumento na macroporosidade pode induzir um fluxo preferencial e contribuir para aumentar as taxas de infiltração (SHIPITALO \& PROTZ, 1987).

$\mathrm{Na}$ transição entre o sistema convencional para o sistema de plantio direto, vários fatores influenciam na qualidade do solo. Entre esses fatores, está a distribuição de poros, cuja avaliação permite qualificar e quantificar os poros existentes em um solo e a eficiência do sistema, consequientemente. A importância dessa distribuição e a influência do manejo do solo têm sido tema de vários estudos (LAL, 1974; STONE \& SILVEIRA, 1999; PRIMAVESI, 1999; BORGES et al., 1999).

Este trabalho teve como objetivo avaliar a distribuição de poros de um Latossolo Vermelho Eutroférrico, como um parâmetro de sua qualidade física para analisar a eficiência da implantação de diferentes sistemas de plantio direto.

\section{MATERIAL E MÉTODOS}

O ensaio foi realizado em Londrina - PR, em um Latossolo Vermelho Eutroférrico, com teores de argila, silte e areia próximos de 620; 150 e $230 \mathrm{~g} \mathrm{~kg}^{-1}$, respectivamente, caracterizando-se, assim, como um solo muito argiloso.

Com latitude de $23^{\circ} 18^{\prime} \mathrm{S}$, longitude de $51^{\circ} 11^{\prime} \mathrm{W}$ e altitude de $566 \mathrm{~m}$, o clima predominante, segundo Wilhem-Köeppen, é do tipo subtropical, sendo a temperatura média do mês mais quente superior a $22{ }^{\circ} \mathrm{C}$ e a do mês mais frio inferior a $18{ }^{\circ} \mathrm{C}$ (CORRÊA et al., 1982).

$\mathrm{O}$ ensaio foi conduzido em faixas de $100 \mathrm{~m}$ de comprimento por $16 \mathrm{~m}$ de largura. No verão, foram semeados milho e soja, alternadamente na área toda. Os tratamentos foram aplicados no inverno, sendo (1) milho safrinha; (2) coquetel (mistura de adubos verdes com aveia-preta, nabo pivotante e ervilhaca); (3) aveia-preta, e (4) trigo. 
O experimento iniciou-se em 1999, com a uniformização do terreno por meio de escarificação. Em seguida, implantou-se a cultura de soja na safra de verão (1999-2000). Na safra de inverno (2000), cada parcela recebeu o tratamento especificado, com exceção do tratamento coquetel, que ficou em pousio. As análises foram feitas no terceiro ano após a implantação do sistema, na safra de verão, quando a cultura implantada em todos os tratamentos foi o milho.

As amostras para a determinação da matéria orgânica (método do carbono) foram coletadas no sistema de tradagem manual, com trado de rosca, nas profundidades 0-0,2 e 0,2-0,4 m, formando uma amostra composta de dez repetições para cada tratamento. Para as mesmas profundidades, retiraram-se amostras, com três repetições, utilizando anéis volumétricos de volume conhecido, para a obtenção dos dados de densidade do solo. As análises de macro e microporosidade e porosidade total foram feitas no Laboratório de Solos da Universidade Estadual de Londrina, pelos métodos de mesa de tensão, nas tensões 0,003 $\mathrm{MPa}$ (30 cm de coluna de água), e 0,006 MPa (60 cm de coluna de água) e no aparelho extrator de Richards, nas tensões 0,01; 0,06 e 1,5 MPa (EMBRAPA, 1997). Os resultados foram submetidos à equação de GENUCHTEN (1980), mediante o programa computacional elaborado por DOURADO NETO et al. (1990), para a obtenção da curva de retenção de água no solo.

O modelo estatístico utilizado foi inteiramente casualizado, com parcela subdividida, sendo os tratamentos os manejos e a profundidade as subparcelas. Efetuou-se a análise de variância, completada pelo teste de Tukey, a 5\% de significância. Essas análises foram processadas por meio do pacote estatístico "Statistical Analysis System" (SAS, 1991).

\section{RESULTADOS E DISCUSSÃO}

Os valores observados na Tabela 1 mostram que, para a profundidade $0-0,2 \mathrm{~m}$, o tratamento milho safrinha obteve o maior valor de matéria orgânica $\left(24,88 \mathrm{~g} \mathrm{~kg}^{-1}\right)$ seguido dos tratamentos coquetel, aveia e trigo $\left(21,11 ; 20,31\right.$ e $\left.17,34 \mathrm{~g} \mathrm{~kg}^{-1}\right)$, respectivamente. Para a profundidade de $0,2-$ 0,4 m, o tratamento milho safrinha manteve o valor de matéria orgânica para essa profundidade, próximo ao valor encontrado nos $0,2 \mathrm{~m}$ iniciais $\left(23,34 \mathrm{~g} \mathrm{~kg}^{-1}\right)$; para os demais tratamentos, esses valores foram inferiores, mantendo-se próximos de $13,5 \mathrm{~g} \mathrm{~kg}^{-1}$ para os tratamentos coquetel e trigo, e $12,05 \mathrm{~g} \mathrm{~kg}^{-1}$ para o tratamento aveia.

TABELA 1. Valores de matéria orgânica $\left(\mathrm{g} \mathrm{kg}^{-1}\right)$ nas profundidades de 0-0,2 $\mathrm{m}$ e 0,2-0,4 m, em função dos tratamentos.

\begin{tabular}{ccccc}
\hline \multirow{2}{*}{ Profundidade $(\mathrm{m})$} & \multicolumn{4}{c}{ Matéria Orgânica $\left(\mathrm{g} \mathrm{kg}^{-1}\right)$} \\
\cline { 2 - 5 } & Milho & Coquetel & Aveia & Trigo \\
\hline $0-0,2$ & 24,88 & 21,11 & 20,31 & 17,34 \\
$0,2-0,4$ & 23,37 & 13,57 & 12,06 & 13,56 \\
\hline
\end{tabular}

Analisando-se as curvas de retenção de água ilustradas na Figura 1, observou-se que os tratamentos afetaram a retenção de água em todas as tensões aplicadas para ambas as profundidades. Para a camada superficial (0-0,2 m), verificou-se que os tratamentos milho safrinha, coquetel e aveia apresentaram mesma tendência da curva para as várias tensões aplicadas, porém o trigo comportou-se de forma diferente, retendo menor quantidade de água em baixas tensões. Esse fato pode ser evidenciado pelos valores de densidade do solo e macroporosidade a $60 \mathrm{cca}$, expressos nas Figuras $2 \mathrm{e}$ 3b, mostrando, apesar de não ter diferido estatisticamente, os maiores valores de densidade e, conseqüentemente, os menores de macroporosidade nessa profundidade. 
(a)

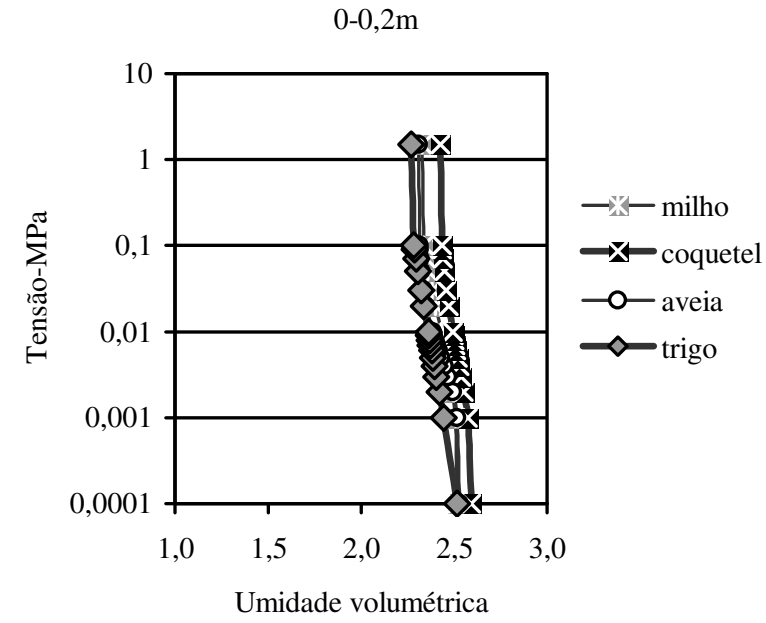

(b)

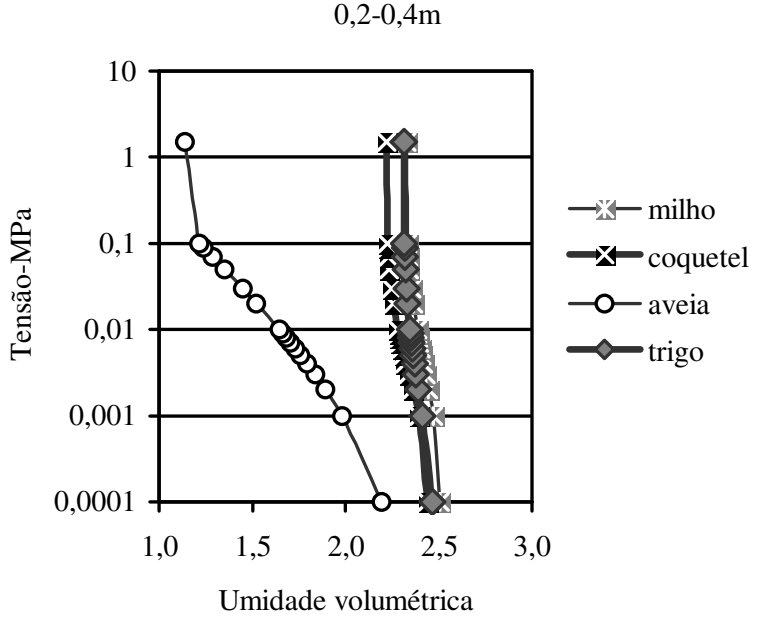

FIGURA 1. Curvas de retenção de água no solo nas profundidades de 0-0,2 m (a) e 0,2-0,4 m (b), em função dos tratamentos.

A curva de retenção de água do tratamento "aveia", na profundidade de 0,2-0,4 m, apresentou um comportamento diferente dos outros tratamentos. Considerando os resultados obtidos por TAVARES FILHO \& TESSIER (1998), trabalhando com solos argilosos, esse formato de curva representa uma distribuição diferente de poros, que podem ter sido afetados por diversos fatores, além da sucessão de culturas.

A quantidade de água retida com alto potencial de água no solo dependeu, primeiramente, da distribuição do tamanho dos poros, sendo afetada pelas estruturas do solo, principalmente a densidade do solo, como observado por RAWLS et al. (1991). À profundidade de 0,2 a 0,4 m, o tratamento aveia diferiu dos outros tratamentos avaliados, apresentando menor retenção de água em todas as tensões submetidas, apesar de os valores de macroporos e das densidades do solo obtidos não terem diferido estatisticamente. Observando as tendências das curvas sob os sistemas de manejo, notou-se que o trigo, o milho e o coquetel apresentaram o mesmo comportamento.

Discordando de BORGES et al. (1999) que, analisando o efeito de níveis de compactação e sua influência nas propriedades físicas de um Latossolo Vermelho-Escuro, observaram um aumento da compactação, causando aumento na quantidade de poros ocupados por água, este trabalho demonstrou que a água retida nos poros, na camada superficial (Figura 1a), não foi influenciada pela densidade do solo (Figura 2). Isso ocorreu apenas no tratamento aveia, na profundidade de 0,2-0,4 m (Figura 1b). Na Figura 2, observa-se que, em todos os tratamentos, os valores de densidade do solo foram significativamente superiores na superfície.

De acordo com GRABLE \& SIEMER (1968), o limite crítico de aeração considerado impeditivo para o crescimento de raízes é de $10 \%$ para culturas anuais. Os valores relativos de macroporosidade dos tratamentos observados nas Figuras 3a e 3b são todos superiores ao limite crítico considerado, podendo-se concluir que, apesar do curto período de manejo, esse já apresenta distribuição de poros adequada para o desenvolvimento de raízes, apesar dos altos valores de densidade do solo. Diversos autores demonstram que, quanto maior a densidade de macroporos, mais o solo pode ser explorado pelas plantas (TIMLIM et al., 1994; LONGSDON et al., 1993; SCOOT et al., 1988). 


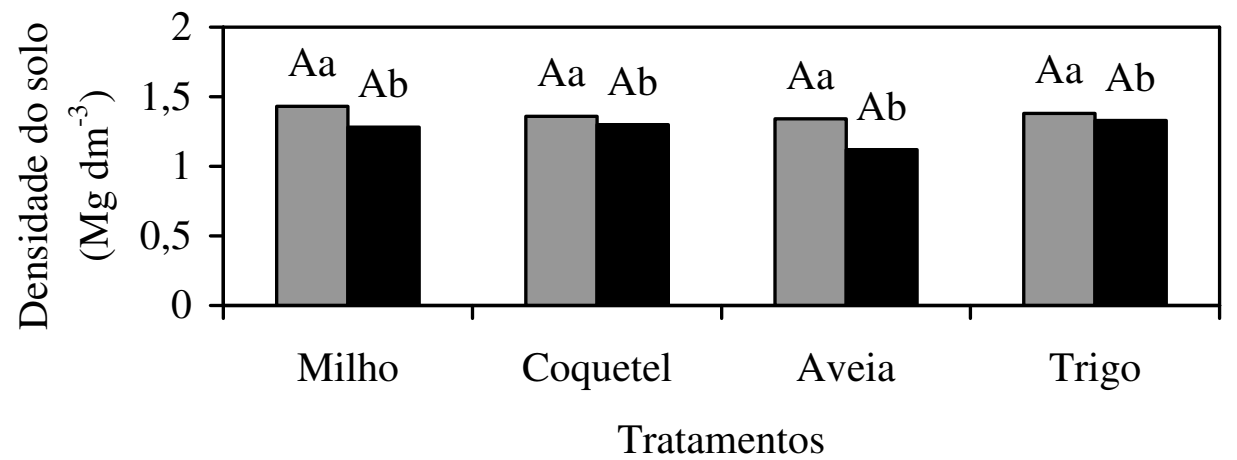

$\square 0-0,2 \mathrm{~m} \square 0,2-0,4 \mathrm{~m}$

* Mesma letra, maiúscula para tratamento e minúscula para profundidade, não difere estatisticamente, a 5\%, pelo teste de Tukey.

FIGURA 2. Densidade do solo nas profundidades de 0-0,2 m e 0,2-0,4 m, em função dos tratamentos.

(a)

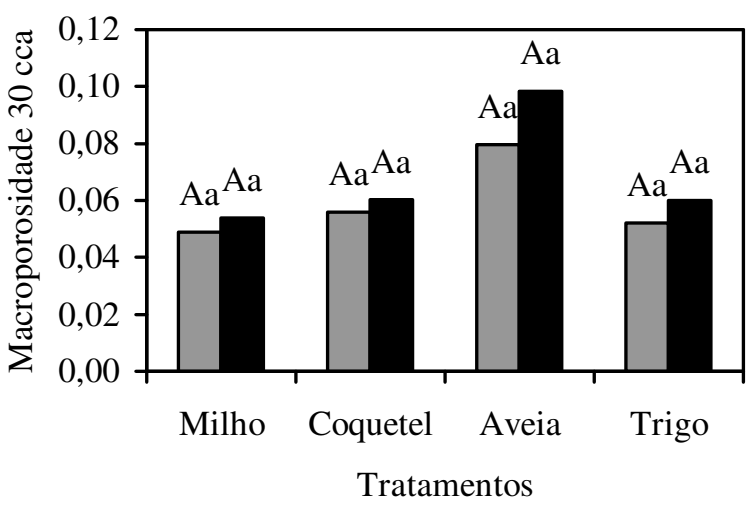

$\square 0-0,2 \mathrm{~m} \square 0,2-0,4 \mathrm{~m}$ (b)

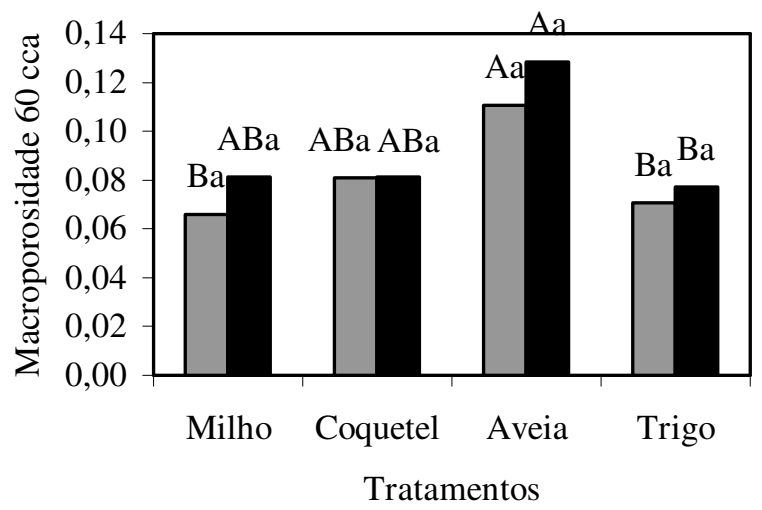

$\square 0-0,2 \mathrm{~m} \square 0,2-0,4 \mathrm{~m}$

* Mesma letra, maiúscula para tratamento e minúscula para profundidade, não difere estatisticamente, a 5\%, pelo teste de Tukey.

FIGURA 3. Macroporosidade do solo entre 0 e $30 \mathrm{cca}$, nas profundidades de 0-0,2 m e 0,2-0,4 m, para os tratamentos (a). Macroporosidade do solo entre 0 e $60 \mathrm{cca}$, nas profundidades de 0-0,2 m e 0,2-0,4 m, para os tratamentos (b).

Apesar de diferir estatisticamente apenas dos tratamentos milho e trigo, na profundidade de 0-0,2 m, e do tratamento trigo, na profundidade de 0,2-0,4 m, os dados da Figura 3 apresentam tendência de aumento da macroporosidade pelo tratamento aveia, nas duas profundidades.

O solo ideal é aquele que contém 1/3 de macroporos (34\%) e 2/3 de microporos (66\%), segundo KIEHL (1979). Considerando a relação da microporosidade nos quatro tratamentos, em ambas as profundidades, pode-se constatar que o solo se encontra numa situação próxima da ideal, refletindo uma capacidade de retenção de água adequada (Figuras 1a e 1b; 3a; 3b; 4, e 5).

Apesar de todos os tratamentos terem apresentado densidades do solo significativamente superiores na camada superficial (Figura 2), o mesmo comportamento não se repete com os dados de 
macro e microporosidade e porosidade total, demonstrando não haver relação direta entre estas características.

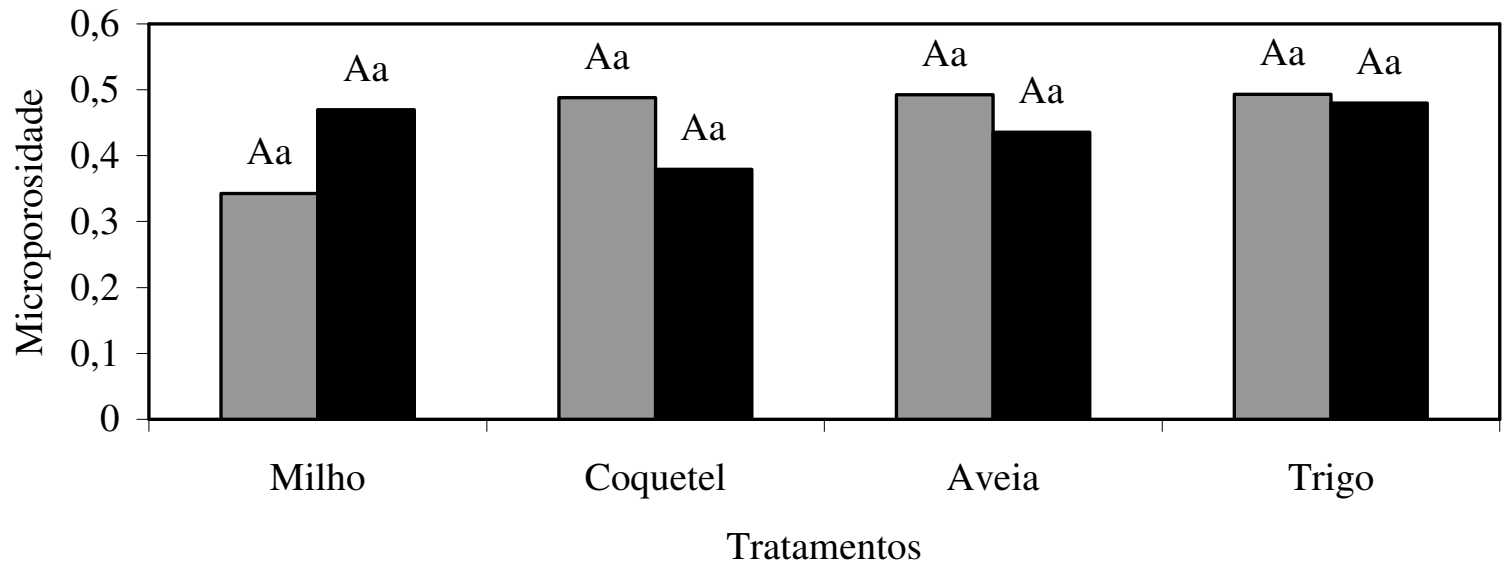

$\square 0-0,2 \mathrm{~m} \square 0,2-0,4 \mathrm{~m}$

* Mesma letra, maiúscula para tratamento e minúscula para profundidade, não difere estatisticamente, a 5\%, pelo Teste de Tukey.

FIGURA 4. Microporosidade do solo nas profundidades de 0-0,2 $\mathrm{m}$ e 0,2-0,4 $\mathrm{m}$, para os quatro tratamentos.

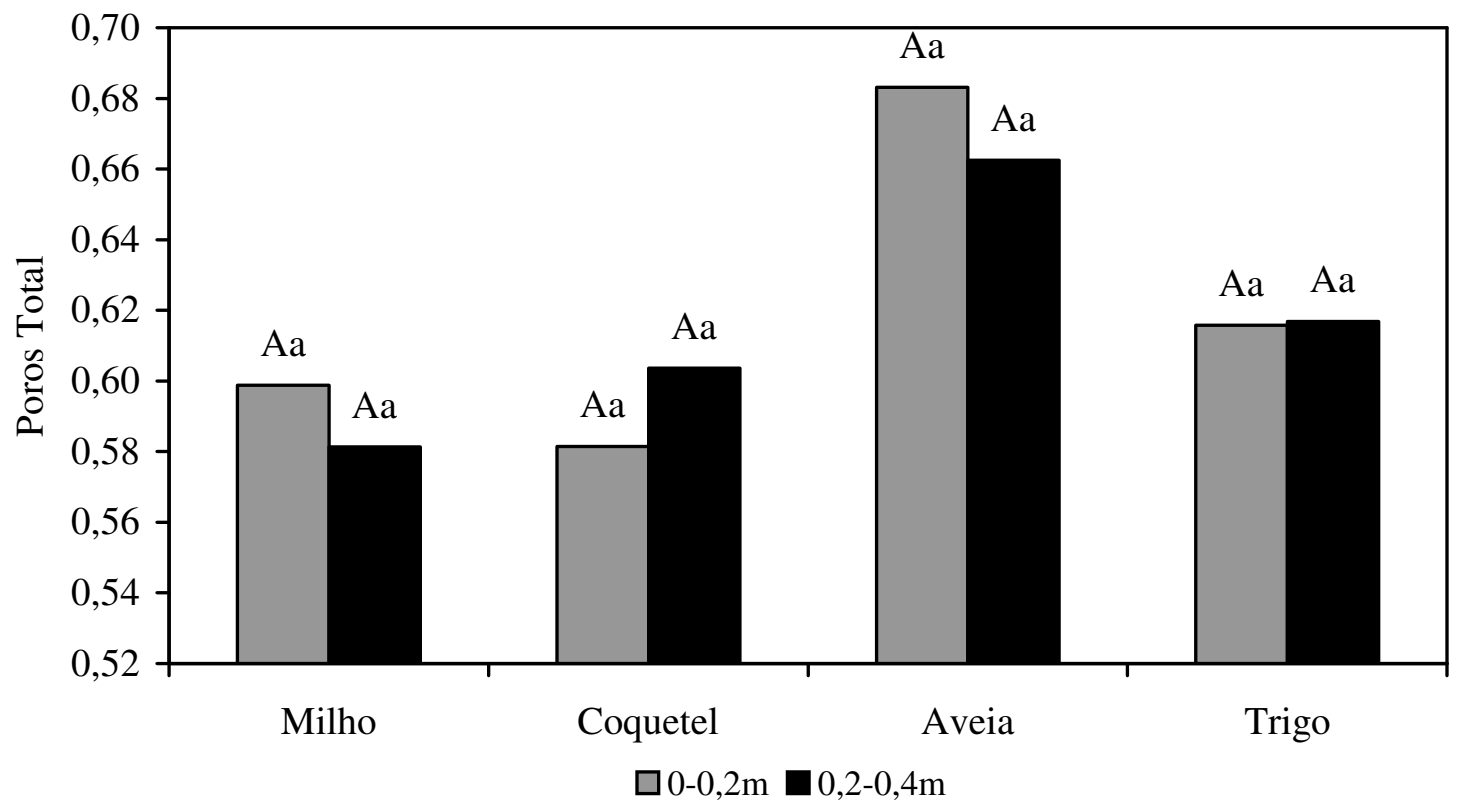

* Mesma letra, maiúscula para tratamento e minúscula para profundidade, não difere estatisticamente, a 5\%, peloTeste de Tukey.

FIGURA 5. Porosidade total do solo, nas profundidades de 0-0,2 $\mathrm{m}$ e $0,2-0,4 \mathrm{~m}$, para os quatro tratamentos. 


\section{CONCLUSÕES}

A utilização de milho safrinha, coquetel de adubos verdes, aveia-preta e trigo, na rotação de inverno, não influenciou na macroporosidade do solo (entre 0 e $30 \mathrm{cca}$ ), na microporosidade, na porosidade total, na retenção de água e na densidade do solo, nas camadas de 0-0,2 e 0,2-0,4 m.

A rotação de inverno com aveia-preta promoveu maior macroporosidade do solo (entre 0 e 60 cca) em relação à rotação com milho e trigo na camada de $0-0,2 \mathrm{~m}$, e com trigo na camada de 0,2-0,4 m.

Para todas as rotações de inverno, os maiores valores de densidade do solo ocorrem na camada de $0-0,2 \mathrm{~m}$.

\section{REFERÊNCIAS BIBLIOGRÁFICAS}

BORGES, E.N.; L. NETO, F.; CORREA, G.F.; BORGES, E.V.S. Alterações físicas introduzidas por diferentes níveis de compactação em Latossolo Vermelho-Escuro textura média. Pesquisa Agropecuária Brasileira, Brasília, v.34, n.9, p.1663-7, 1999.

CORREAA, A.R.; GODOY, H.; BERNARDES, R.L.M. Características climáticas de Londrina. 2.ed. Londrina: IAPAR, 1982. 16 p. (Circular, 5)

CORSINI, P.C.; FERRAUDO, A.S. Efeitos de sistemas de cultivo na densidade e macroporosidade do solo e no desenvolvimento radicular do milho em latossolo roxo. Pesquisa Agropecuária Brasileira, Brasília, v.34, n.32, p.289-98, 1999.

DOURADO NETO, D.; JONG-VAN-LIES, Q.; BOTREL, T.A.; LIBARDI, P.L. Programa para confecção de curva de retenção de água no solo, utilizando o modelo de Genuchten. Engenharia Rural, Piracicaba, v.1, n.2, p.92-202, 1990.

EMBRAPA - Centro Nacional de Pesquisa de Solos. Manual de métodos e análises de solo. 2.ed. rev. Rio de Janeiro, 1997. 255 p.

GENUCHTEN, M. van. A closed-form equation for predicting the hydraulic condutivity of unsatured soil. Soil Science Society of American Journal, Madison, v.1, p.892-8, 1980.

GRABLE, A.R.; SIEMER, E.F. Effects of bulk density, aggregate size and soil water suction on oxygen diffusion, redox potential and elongation of corn roots. Soil Science Society of American Journal, Madison, v.32, p.180-6, 1968.

HILLEL, D. Introduction to soil physics. London: Academic Press, 1982. 364 p.

HORTON, R.; ANKENY, M.D.; ALLMARAS, R.R. Effects of soil compactation on soil hydraulic porperties. In: SOANE, B.D.; OUWERKERK, C. van (Ed.). Soil compactation in crop production. Amsterdam: Elsevier, 1994. p.141-65.

JORGE, J.A.; PRADO, H. do. Porosidade, consistência e propriedades hídricas de três podzólicos vermelho-amarelos e uma terra roxa estruturada. Revista Brasileira de Ciência do Solo, Viçosa, v.12, p.1-6, 1988.

KIEHL, E.J. Manual de edafologia. São Paulo: Agronômica Ceres, 1979. 262 p.

LAL, R. No-tillage effects on soil properties and maize (Zea mays L.) production in Western Nigeria. Plant and Soil, Amsterdam, v.40, p.321-31, 1974.

LONGSDON, S.D.; McCOY, E.L.; ALLMARAS, R.R.; LINDEN, D.R. Macropores characterization by indirect methods. Journal of Soil Science, Baltimore, v.155, p.316-24, 1993.

PRIMAVESI, A. Manejo ecológico do solo. São Paulo: Editora Nobel, 1999. 549 p. 
RALWS, W.J.; GISH, T.J.; BRAKENSIEK, D.L. Estimating soil water retention from soil physical properties and characteristics. Advances in Soil Science, New York, v.16, p.213-34, 1991.

SAS INSTITUTE. SAS/STAT procedure guide for personal computers. Version 5. Cary: SAS Inst., 1991. $1.686 \mathrm{p}$.

SCOOT, G.J.T.; WEBSTER, R.; NORTICLIFF, S. The topology of pores structure in cracking clay soil. Journal of Soil Science, Baltimore, v.39, p.303-14, 1988.

SHIPITALO, M.J.; PROTZ, R. Comparison of morphology and porosity of a soil under conventional and zero-tillage. Canadian Journal of Soil Science, Ottawa, v.67, p.445-56, 1987.

STONE, L.F.; SILVEIRA, P.D. Efeito do sistema de preparo na compactação do solo, disponibilidade hídrica e comportamento do feijoeiro. Pesquisa Agropecuária Brasileira, Brasília, v.34, n.1, p.83-91, 1999.

TAVARES FILHO, J. ; TESSIER, D. Influence de pratiques culturales sur le comportement et les propriétés de sols du Paraná (Brésil). Étude et Gestion des Sols, Orleans, v.5, p.61-71, 1998.

TIMLIM, D.J.; AHUJA, L.R.; ANKENY, M.D. Comparison of three field methods to characterize apparent macro pore conductivity. Soil Science Society of American Journal, Madison, v.58, p.278-84, 1994. 